\title{
Multimodal quantitative magnetic resonance imaging for lumbar intervertebral disc degeneration
}

\author{
WEI WANG ${ }^{1-3^{*}}$, JIN HOU $^{4 *}$, DEYONG LV ${ }^{5}$, WEN LIANG $^{1}$, XINQING JIANG ${ }^{2}$, \\ HONGBIN HAN $^{3}$ and XIANYUE QUAN ${ }^{1}$ \\ ${ }^{1}$ Department of Radiology, Zhujiang Hospital, Southern Medical University, Guangzhou, Guangdong 510280; \\ ${ }^{2}$ Department of Radiology, Guangzhou First People's Hospital, Guangzhou, Guangdong 510180; \\ ${ }^{3}$ Department of Radiology and Beijing MRI Technology Research Laboratory, Peking University Third Hospital, \\ Beijing 100191; ' Department of Radiology, The Second Affiliated Hospital of Guangzhou Medical University, \\ Guangzhou, Guangdong 510260; ${ }^{5}$ Department of Radiology, Dongying People's Hospital of Shandong, \\ Dongying, Shandong 257091, P.R. China
}

Received March 11, 2016; Accepted March 17, 2017

DOI: $10.3892 /$ etm.2017.4786

\begin{abstract}
The present study investigated the application of the $\mathrm{T}_{1 \rho}$ and $\mathrm{T}_{2}$ relaxation mapping magnetic resonance imaging (MRI) and diffusion-weighted imaging (DWI) in the evaluation of intervertebral disc degeneration (IDD). A total of 93 asymptomatic subjects were imaged with $\mathrm{T}_{1 \rho}$ and $\mathrm{T}_{2}$ mapping, as well as DWI. Pfirrmann grading was performed and correlation analysis was conducted for $\mathrm{T}_{1 \rho}, \mathrm{T}_{2}$ and DWI results with the grading results and age. Pfirrmann grading indicated 69 cases of grade I, 240 cases of grade II, 101 cases of grade III, 43 cases of grade IV and 12 cases of grade V. MRI showed that the $T_{1 \rho}$ values of the nucleus pulposi at L4/5 and L5/S1 were significantly reduced $(\mathrm{P}<0.05)$ and no significant differences were observed in the $\mathrm{T}_{2}$ values compared with the values of the nucleus pulposus at L1/2, L2/3 and L3/4. The apparent diffusion coefficient (ADC) values of L1/2 were significantly decreased from L2/3 and L3/4 $(\mathrm{P}<0.05)$. Correlation analysis revealed that the $T_{1 \rho}, T$, and $A D C$ values were positively correlated with each other. Moreover, the $\mathrm{T}_{1 \rho}$ values were significantly decreased with the increase of Pfirrmann grades
\end{abstract}

Correspondence to: Dr Hongbin Han, Department of Radiology and Beijing MRI Technology Research Laboratory, Peking University Third Hospital, 49 Huayuanbei Road, Beijing 100191, P.R. China

E-mail: hanhongbin@126.com

Dr Xianyue Quan, Department of Radiology, Zhujiang Hospital, Southern Medical University, 253 Industrial Road, Guangzhou, Guangdong 510280, P.R. China

E-mail: quanxianyue63@163.com

*Contributed equally

Key words: intervertebral disc degeneration, magnetic resonance imaging, $\mathrm{T}_{1 \rho}, \mathrm{T}_{2}$, diffusion-weighted imaging
$(\mathrm{P}<0.05)$, with the exception of grades IV and V. However, $\mathrm{T}_{2}$ and ADC values were not significantly different between grades I and II or IV and V. In addition, the $\mathrm{T}_{1 \rho}, \mathrm{T}_{2}$ and ADC values were significantly decreased with the increase of age in patients with IDD $(\mathrm{P}<0.05) . \mathrm{T}_{1 \rho}$ and $\mathrm{T}_{2}$ mapping and DWI are promising techniques for the in vivo diagnosis of IDD, which may be useful in determining the appropriate prevention and treatment options for the disease.

\section{Introduction}

Lower back pain (LBP) affects $70-85 \%$ of adults during their lifetime and approximately $45 \%$ of LBP cases appear to be of the discogenic origin (1). One of the underlying mechanisms of symptomatic disc diseases is intervertebral disc degeneration (IDD), which has also been shown to be associated with the pathogenesis of $\operatorname{LBP}(2,3)$. However, it is still difficult to distinguish between normal aging and pathological degeneration in the detection and diagnosis of IDD, making it unfavorable for the timely and adequate treatment of this disease $(4,5)$.

Due to its advantages, including no-ionization damage, excellent soft-tissue contrast and multi-view imaging, magnetic resonance imaging (MRI) has been widely used in non-invasive detection or IDD and several classification systems have been established based on the structure, morphology and signal intensity of discs in IDD (6,7). The Pfirrmann scoring system provides a semi-quantitative assessment of IDD in vivo, whereas $\mathrm{T}_{2}$-weighted imaging $\left(\mathrm{T}_{2} \mathrm{WI}\right)$-based grading systems are particularly suitable for establishing the degeneration in later stages (8). However, the application of these scoring systems in evaluating IDD in clinic settings is limited due to the unsatisfactory objectivity.

In recent years, it has been indicated that the $T_{1 \rho}$ technique may aid the detection of IDD due to its sensitivity to the proteoglycan content in discs (9-11). Moreover, the quantitative $\mathrm{T}_{2}$ relaxation time measurement has also been reported to reflect the molecular environment in discs. Several in vivo studies have revealed that the degenerated discs, with reduced 
water content and decreased structural integrity, exhibit lower $\mathrm{T}_{2}$ values than normal discs, which is correlated with the Pfirrmann grading (12-16). Furthermore, the apparent diffusion coefficient (ADC) from diffusion-weighted imaging (DWI) is another potential indicator for the detection of IDD, which indirectly reflects alterations in nucleus pulposi matrix content (17). Significantly decreased ADC values have been reported in degenerated nucleus pulposi compared with normal discs $(18,19)$. Few studies have evaluated and compared the performance of $T_{1 \rho}$ and $T_{2}$ relaxation time measurements and the DWI technique in the assessment of IDD $(20,21)$.

In the present study, the application of the $T_{1 \rho}$ and $T_{2}$ quantitative MRI and the DWI technique were utilized for the evaluation of IDD and compared. Asymptomatic volunteers were imaged with $\mathrm{T}_{1 \rho}$ and $\mathrm{T}_{2}$ mapping, as well as DWI, and subjected to the Pfirrmann grading. Correlation analysis was conducted for the $T_{1 \rho}, T_{2}$ and DWI results with Pfirrmann grading and age.

\section{Materials and methods}

Study subjects. A total of 93 asymptomatic subjects, 38 males and 55 females, with an average age of $34.2 \pm 14.0$ years (ranging from 20 to 76 years), were recruited in the present study. The inclusion criteria were: i) No recent history (within 6 months) of weight-bearing work; ii) no significant back ache with in the past 6 months; and iii) no history of spine trauma and spinal deformity.

All the subjects underwent $\mathrm{T}_{1 \rho}$ and $\mathrm{T}_{2}$ imaging, while additional DWI was performed on 47 among them (18 males and 29 females; with an average age of $39.0 \pm 13.5$ years, ranging from 22 to 63 years). The investigation was conducted in the evening (between 5:00 and 10:00 p.m.). Before scanning, the subjects were advised not to perform strenuous exercise and were encouraged to lie down for $\sim 30 \mathrm{~min}$ to minimize the diurnal variation during the $\mathrm{T}_{2}$ and ADC measurements of the intervertebral discs. Prior written and informed consent was obtained from each patient and the study was approved by the Ethics Review Board of the Human Research Ethics Committee at Zhujiang Hospital (Guangzhou, China).

MRI. MRI was performed using a 3.0T whole-body multitransmit scanner system (Achieva TX; Philips Healthcare, DA Best, The Netherlands), with a dedicated 6-channel spine coil. For the Pfirrmann grading, sagittal $\mathrm{T}_{2}$-weighted imaging $\left(\mathrm{T}_{2} \mathrm{WI}\right)$ was acquired twice using a fast spin echo sequence with the following parameters: Repetition time (TR), 3,000 msec; echo time (TE), $128 \mathrm{msec}$; slice thickness, $5 \mathrm{~mm}$; matrix, 448x448 and 288x288; field of view (FOV), 220x201 mm and 180x56 mm, respectively. Sagittal $\mathrm{T}_{1 \mathrm{p}}$ scanning was performed using a 3D steady gradient echo sequence with the following parameters: TR, $4.85 \mathrm{msec}$; TE, 2.39 msec; spin-lock frequency, $500 \mathrm{~Hz}$; spin-lock time, 0/10/20/30/40 msec; flip angle, $50^{\circ}$; slice thickness, $5 \mathrm{~mm}$; matrix, 448x448; FOV, 220x201 mm. Sagittal $\mathrm{T}_{2}$ mapping was performed with the following parameters: TR, 1,162 msec; TE, 20-100 msec; number of echoes, 5; slice thickness, $5 \mathrm{~mm}$; matrix, 448x448; FOV, 220x201 mm. DWI was conducted with the following parameters: TR, 3,000 msec; TE, $64 \mathrm{msec}$; b-values, 0 and $500 \mathrm{~mm}^{2} / \mathrm{sec}$; slice thickness, $5 \mathrm{~mm}$; matrix, 288x288; FOV, 180x56 mm. Region of interest (ROI) analysis was performed on midsagittal $\mathrm{T}_{2} \mathrm{WI}$ (Fig. 1).

Pfirrmann grading. Based on the MRI results, Pfirrmann grading of lumbar discs was performed by two independent radiologists (each with $>10$ years of experience in imaging), according to the grading system described by Griffith et al (7). Five lumbar intervertebral discs (L1-S1) were analyzed and the grading results were recorded for analysis if the consensus was reached with the following criteria: Normal, grade I; mild degeneration, grade II; moderate degeneration, grade III; and severe degeneration, grades IV and V.

Inter- and intra-observer reliability analysis. Inter-and intra-observer reliabilities of Pfirrmann grading were examined using the kappa concordance test with the following criteria: Slight agreement, 0 to 0.2 ; fair agreement, 0.21 to 0.4 ; moderate agreement, 0.41 to 0.60 ; substantial agreement, 0.61 to 0.8 ; and excellent agreement, 0.81 .

Statistical analysis. SPSS 15.0 software (SPSS, Inc., Chicago, IL, USA) was used for statistical analysis. Pfirrmann grading results were compared with analysis of variance, underwent Bonferroni post hoc tests and were subjected to the receiver operating curve (ROC) analysis. Spearman and Pearson correlation coefficients were determined for the quantitative values. $\mathrm{P}<0.05$ was considered to indicate a statistically significant difference.

\section{Results}

Pfirrmann grading of lumbar intervertebral discs. A total of 465 lumbar intervertebral discs were subjected to Pfirrmann grading by two independent radiologists. Results of the inter- and intra-observer reliability analysis are summarized in Table I. Excellent agreement was obtained from the radiologists, with the $\kappa$ values ranging from 0.886 to 0.938 . Disagreement in grading maybe due to the difficulties in identifying structures that exhibited homogeneity, such as distinguishing between grade I and II and distinguishing between the annulus and nucleus (grade II-III).

The Pfirrmann grading results indicated that, 69 cases were classified as Pfirrmann grade I, 240 cases as grade II, 101 cases as grade III, 43 cases as grade IV, and 12 cases as grade V. Eventually $\mathrm{T}_{1 \rho}$ values of 383 discs, $\mathrm{T}_{2}$ values of 445 discs and ADC values of 231 discs were successfully obtained. Subsequently, the correlations between the $\mathrm{T}_{1 \rho}, \mathrm{T}_{2}$ and ADC values with Pfirrmann grading and age were analyzed.

Correlation analysis between $T_{1 \rho}, T_{2}$, and ADC values with Pfirmann grading. MRI indicated that the $\mathrm{T}_{1 \rho}$ values of nucleus pulposi at L4/5 and L5/S1 were significantly reduced compared with the values of the nucleus pulposus at L1/2, L2/3 and L3/4 $(\mathrm{P}<0.05)$, whereas no significant differences were observed in the $\mathrm{T}_{2}$ value. In addition, the ADC values of $\mathrm{L} 1 / 2$ were significantly different from the L2/3 and L3/4 $(\mathrm{P}<0.05$; Fig. 2).

Correlation analysis between the $\mathrm{T}_{1 \rho}, \mathrm{T}_{2}$ and ADC values indicated the positive relationship between each other (Fig. 3). 
Table I. Inter- and intra-observer reliability analysis of Pfirrmann grading.

Disagreement

\begin{tabular}{|c|c|c|c|c|c|c|c|c|c|c|c|c|c|}
\hline \multirow[b]{2}{*}{ Variable } & \multirow[b]{2}{*}{ Kappa } & \multicolumn{2}{|c|}{ Agreement } & \multicolumn{2}{|c|}{ I } & \multicolumn{2}{|c|}{ II } & \multicolumn{2}{|c|}{ III } & \multicolumn{2}{|c|}{ IV } & \multicolumn{2}{|c|}{$\mathrm{V}$} \\
\hline & & $\mathrm{n}$ & $\%$ & $\mathrm{n}$ & $\%$ & $\mathrm{n}$ & $\%$ & $\mathrm{n}$ & $\%$ & $\mathrm{n}$ & $\%$ & $\mathrm{n}$ & $\%$ \\
\hline Interobserver reliability & 0.886 & 444 & 95.5 & 8 & 11.6 & 17 & 7.1 & 6 & 5.9 & 0 & 0 & 0 & 0 \\
\hline Intraobserver reliability & & & & & & & & & & & & & \\
\hline Radiologist 1 & 0.938 & 446 & 95.9 & 6 & 8.7 & 7 & 3.9 & 6 & 5.9 & 0 & 0 & 0 & 0 \\
\hline Radiologist 2 & 0.930 & 445 & 95.6 & 9 & 13.0 & 7 & 3.9 & 4 & 3.9 & 0 & 0 & 0 & 0 \\
\hline
\end{tabular}

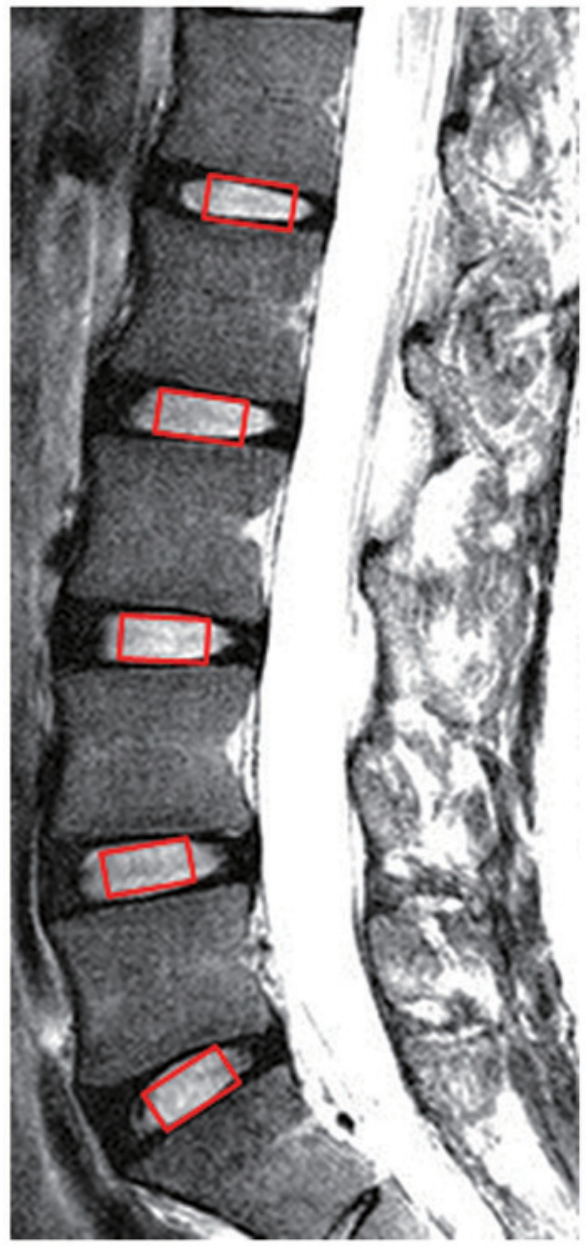

Figure 1. Schematic diagram of regions of interest. Regions of interest were selected as the rectangular areas with equal sizes at each disc level in the center sagittal planes.

Correlation analysis between the $\mathrm{T}_{1 \rho}, \mathrm{T}_{2}$ and $\mathrm{ADC}$ values with Pfirrmann grading revealed that $\mathrm{T}_{1 \rho}$ values were significantly different between the Pfirrmann grades $(\mathrm{P}<0.05$; with the exception of grades IV and $\mathrm{V}$ ) and the $\mathrm{T}_{2}$ and $\mathrm{ADC}$ values were not significantly different between grades I and grades II, as well as grades IV and V (Table II). Moreover, results from the ROC analysis, which indicated the cut-off value, sensitivity and specificity of $T_{1 p}, T_{2}$ and ADC, are shown in Fig. 4 and Table III. In particular, $\mathrm{T}_{2}$ mapping demonstrated the highest accuracy detecting the later degenerative changes.
These results suggest that significantly negative correlations exist between the $T_{1 \rho}, T_{2}$ and ADC values and the Pfirrmann grading.

Correlation analysis between $T_{1 \rho}, T_{2}$ and ADC values with age. Results of the correlation analysis between the $\mathrm{T}_{1 \rho}, \mathrm{T}_{2}$ and ADC values and age are shown in Fig. 5. All the $T_{1 \rho}, T_{2}$ and ADC values were significantly decreased with the increase of age and Spearman correlation coefficients of $-0.349,-0.594$, and-0.387 were demonstrated, respectively (all $\mathrm{P}<0.01$ ). Specifically, $T_{1 \rho}$ values remained relatively stable across the age range of 20-45 years and continuously declined after the age of 45 years. Moreover, $\mathrm{T}_{2}$ values were slowly decreased over the ages of 20-45 years, whereas the values were slightly increased over the age range of 45-50 years. Furthermore, the values were significantly declined after the age of 50 years $(\mathrm{P}<0.01)$. Additionally, the ADC values were slowly decreased over the age range of 45-50 years $(\mathrm{P}<0.05)$. However, the ADC values fell rapidly after the age of 50 years $(\mathrm{P}<0.01)$. These results suggest that the $T_{1 \rho}, T_{2}$ and $A D C$ values were decreased with the increasing of age and exhibited slight variations in changing patterns.

\section{Discussion}

In the $\mathrm{T}_{1 \mathrm{p}}, \mathrm{T}_{2}$ and DWI MRI techniques were applied to assess subjects with IDD at different stages. Subjects with ages ranging from 20 to 76 years old were investigated. Results from the $T_{1 \rho}$ and $\mathrm{T}_{2}$ mapping, as well as DWI from the same sagittal plane were compared and analyzed. Pfirrmann grading was also performed according to a previously published protocol (7). Regarding previous findings $(18,20-22)$, the present study covered unified regions of interests and predominantly focused on the detection of nucleus pulposi in IDD. Color-coded $T_{1 \rho}$ and $\mathrm{T}_{2}$ mapping revealed that the normal nucleus pulposus may be divided into the peripheral low-value and core high-value areas, based on the inside proteoglycan distribution. The dynamic range of $T_{1 \rho}$ mapping was greater than $T_{2}$ mapping, which may affect the measurement sensitivity to early subtle degenerative alterations. However, in the ADC mapping of normal discs, higher detection values were observed in the peripheral area of nucleus pulposi. We suggested that ADC measurements would be influenced by different factors and provide complementary information about the disc composition. The present results indicated that in the degenerated discs attenuated $\mathrm{T}_{2} \mathrm{WI}$ signals 
A
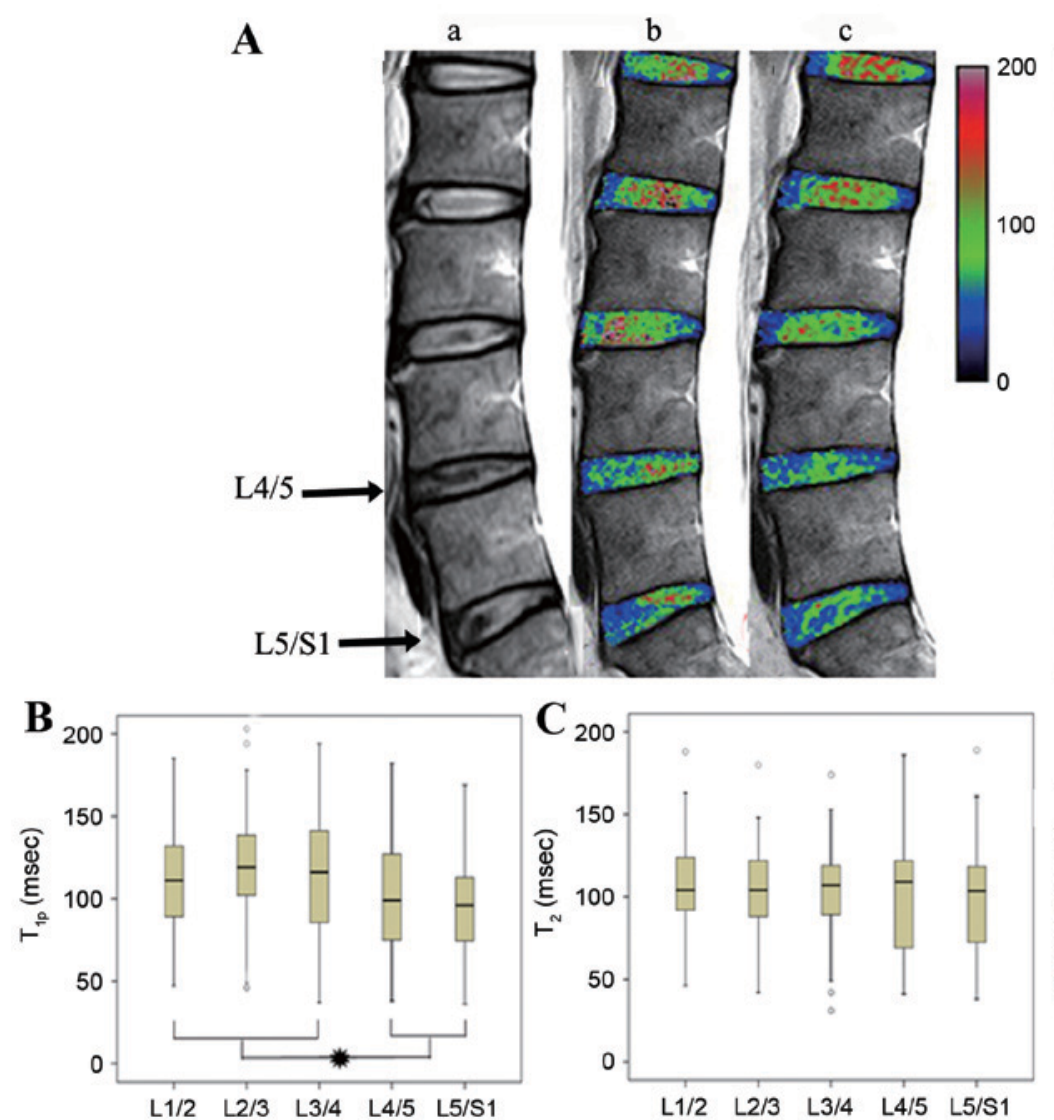

$\mathrm{C}_{200}$

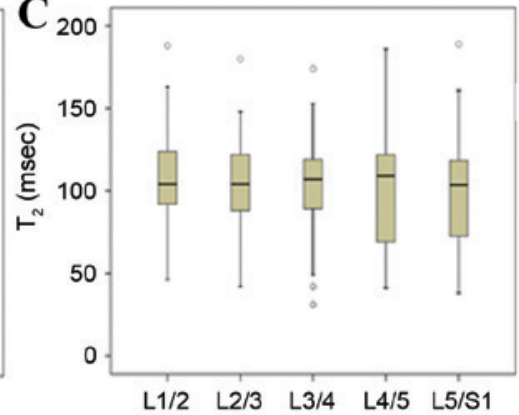

d

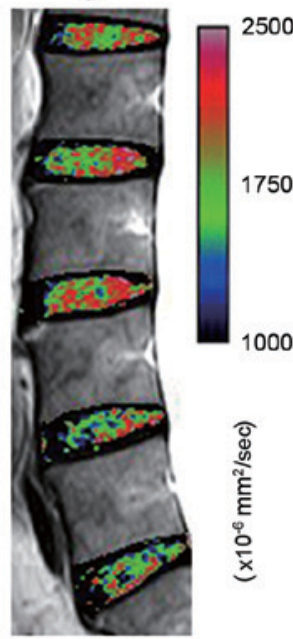

D

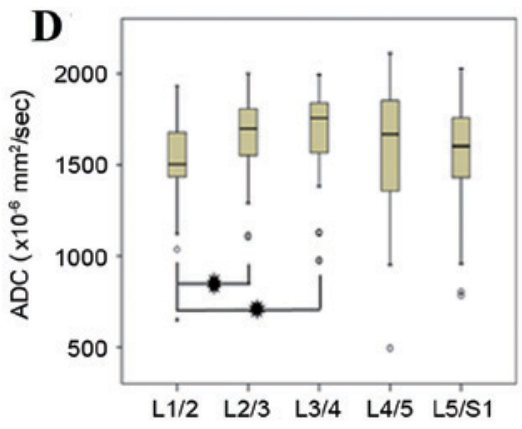

Figure 2. Magnetic resonance imaging results of lumber intervertebral discs. (A) Representative pictures of magnetic resonance imaging. (a) $\mathrm{T}_{2}$-weighted imaging of L4/5 and L5/S1 assessed as grade III, showing the gray nucleus and the unclear distinction of nucleus and annulus, without collapsed disc space (indicated by arrows). Discs at other levels were grade II. The value-based color-scale images displayed the spatial distribution of the (b) $\mathrm{T}_{1}$, (c) $\mathrm{T}_{2}$ and (d) ADC values of discs and were overlaid on the $\mathrm{T}_{2} \mathrm{WI}$ image. As shown by the color scale bar, the values increased from dark to white, that was, blue indicated the lower value and red indicated the higher values. (B) The mean $\mathrm{T}_{1 \rho},(\mathrm{C}) \mathrm{T}_{2}$ and (D) ADC values in magnetic resonance imaging of lumbar intervertebral discs at each level. ${ }^{*} \mathrm{P}<0.05$. ADC, apparent diffusion coefficient.

A

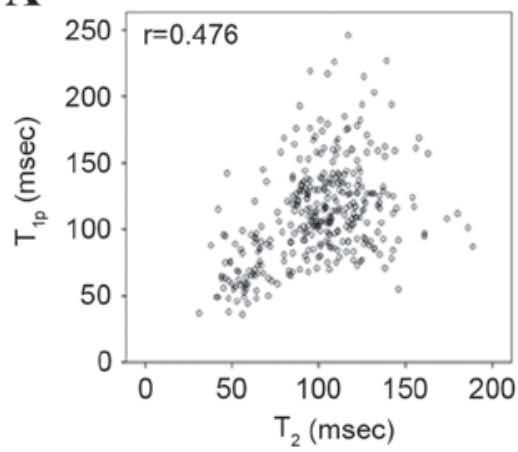

B

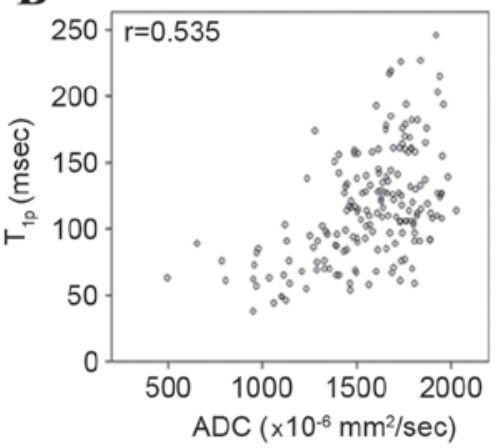

C

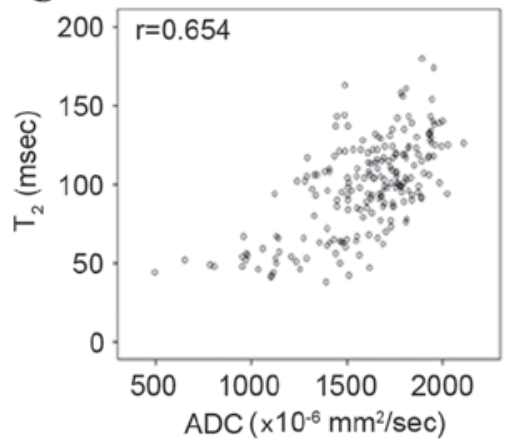

Figure 3. Correlation analysis of magnetic resonance imaging. Scatter plots from the correlation analysis between the (A) $T_{1 \rho}$ and $T_{2}$ values (B), $T_{1 \rho}$ and $A D C$ values and (C) $\mathrm{T}_{2}$ and ADC values in magnetic resonance imaging. ADC, apparent diffusion coefficient.

and the three quantitative measures of nucleus pulposi were decreased when disease severity was increased.

In line with previous findings, the present study demonstrated that $\mathrm{T}_{1 \rho}$ and $\mathrm{T}_{2}$ values were negatively correlated with Pfirrmann grading and tended to decrease as the disease progressed $(18,21-25)$. Notably, $\mathrm{T}_{1 \rho}$ values of the Pfirrmann grade II discs were significantly greater than the Pfirrmann grade $I$ discs, suggesting that the in vivo $\mathrm{T}_{1 \rho}$ quantification may be more sensitive to early macromolecular changes in the matrix of nucleus pulposi. However, the present results from the ROC analysis showed that the $\mathrm{T}_{2}$ quantification revealed the greatest accuracy in detecting the later degenerative changes and the $T_{1 \rho}$ method was associated with the lowest accuracy. This observation may be due to the fact that $\mathrm{T}_{2}$ is highly related to collagen integrity (26) and is not particularly sensitive to the changes of proteoglycan content (25). A previous study has reported that IDD primarily occurs in the lower lumbar region, particularly at L4/L5 and L5/S1 (27). 
Table II. T1 $\rho$, T2, and ADC values of nucleus pulposus with different Pfirrmann grades.

\begin{tabular}{lcccccc}
\hline Parameter & Grade I & Grade II & Grade III & Grade IV & Grade V & Spearman \\
\hline $\mathrm{T}_{1 \rho}(\mathrm{msec})$ & $131.38 \pm 28.30$ & $120.74 \pm 33.49^{\mathrm{a}}$ & $93.24 \pm 30.20^{\mathrm{b}}$ & $68.56 \pm 22.25^{\mathrm{c}}$ & $64.88 \pm 16.54$ & $\mathrm{r}_{\mathrm{s}}=-0.561$ \\
& & & & & & $\mathrm{P}<0.01$ \\
$\mathrm{~T}_{2}(\mathrm{msec})$ & $121.45 \pm 19.15$ & $113.60 \pm 19.75$ & $83.54 \pm 19.13^{\mathrm{b}}$ & $55.26 \pm 10.79^{\mathrm{c}}$ & $59.09 \pm 21.62$ & $\mathrm{r}_{\mathrm{s}}=-0.690$ \\
& & & & & \\
$\mathrm{ADC}\left(\mathrm{x} 10^{-3} \mathrm{~mm}^{2} / \mathrm{sec}\right)$ & $1.764 \pm 0.212$ & $1.692 \pm 0.189$ & $1.564 \pm 0.211^{\mathrm{b}}$ & $1.263 \pm 0.309^{\mathrm{c}}$ & $1.156 \pm 0.392$ & $\begin{array}{l}\mathrm{r}_{\mathrm{s}}=-0.530 \\
\end{array}$ \\
& & & & & & $\mathrm{P}<0.01$ \\
\hline
\end{tabular}

${ }^{\mathrm{a}} \mathrm{P}<0.05$ vs. grade I; ${ }^{\mathrm{b}} \mathrm{P}<0.01$ vs. grade II; ${ }^{\mathrm{c}} \mathrm{P}<0.01$ vs. grade III. ADC, apparent diffusion coefficient.

Table III. Receiver operating curve analysis of magnetic resonance imaging.

\begin{tabular}{lccccc}
\hline Parameter & Pfirrmann grades & Area under curve & Cut-off value & Sensitivity (\%) & Specificity (\%) \\
\hline T1 $\rho$ & I-II & 0.624 & $116.5 \mathrm{msec}$ & 70.0 & 53.6 \\
& II-III & 0.730 & $103.5 \mathrm{msec}$ & 66.8 & 68.6 \\
T2 & III-IV & 0.740 & $86.5 \mathrm{msec}$ & 58.1 & 79.1 \\
& II-III & 0.871 & $94.5 \mathrm{msec}$ & 83.7 & 73.9 \\
Apparent diffusion & III-IV & 0.910 & $67.5 \mathrm{msec}$ & 76.1 & 99.3 \\
coefficient & II-III & 0.670 & $1.732 \times 10^{-3} \mathrm{~mm}^{2} / \mathrm{sec}$ & 48.2 & 81.8 \\
\hline
\end{tabular}

\section{$\mathbf{A}$}

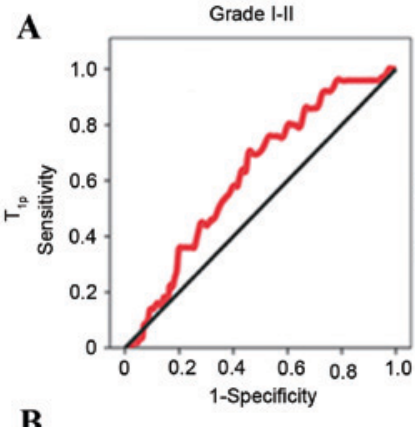

B
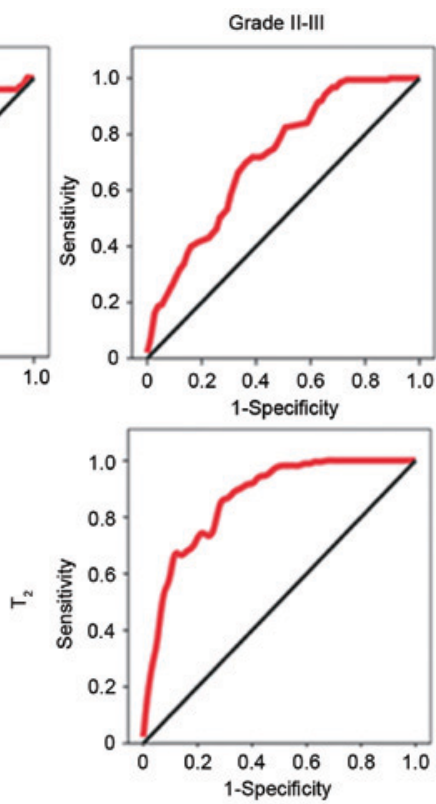

C

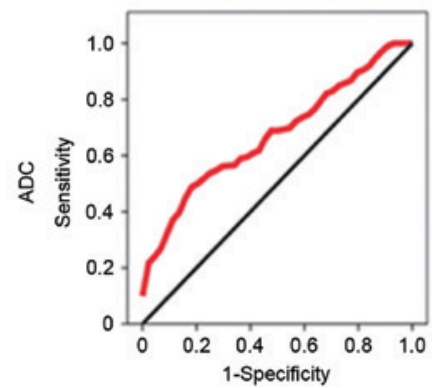

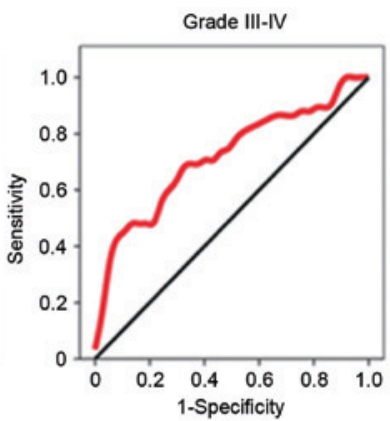
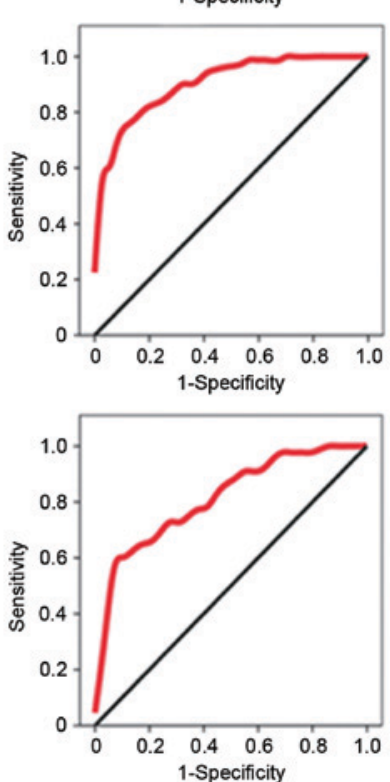

Figure 4. Receiver operating curve analysis of magnetic resonance imaging. Receiver operating curves of (A) $T_{1 p}$, (B) $T_{2}$ and (C) ADC values in diagnosing intervertebral disc degeneration with different Pfirrmann grades (I-IV). ADC, apparent diffusion coefficient. 
A

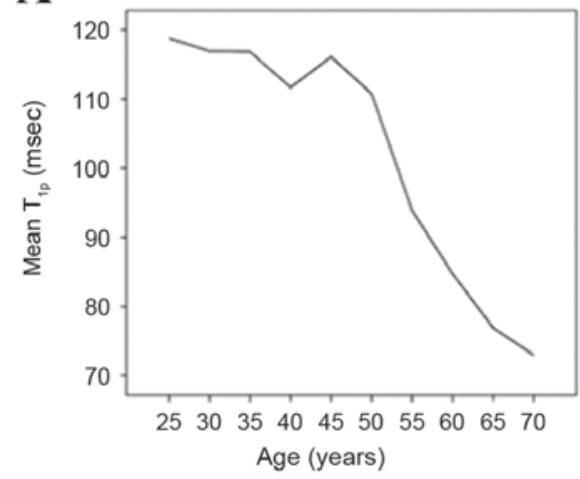

B

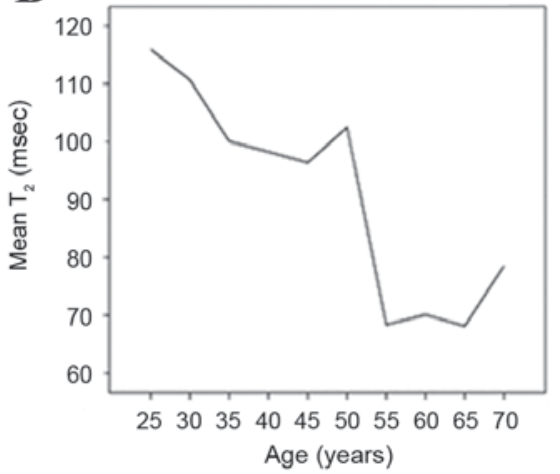

C

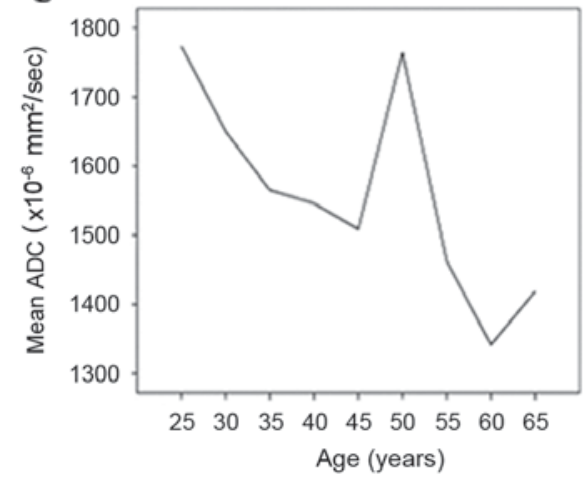

Figure 5. Correlation analysis of $\mathrm{T}_{1 p}, \mathrm{~T}_{2}$, and ADC values with age. (A) Mean $\mathrm{T}_{1 \mathrm{p}},(\mathrm{B}) \mathrm{T}_{2}$ and (C) ADC values of subjects with different ages. ADC, apparent diffusion coefficient.

In the present study, significant differences in the $\mathrm{T}_{1 \rho}$ value were only identified in the lower discs (L4/L5 and L5/S1) compared with the other disc levels, suggesting that $T_{1 \rho}$ may be more sensitive to early IDD pathogenesis. Degenerative changes in the disc matrix are inevitable and begin soon after birth (28). Previous studies have reported various age-related changes in the intervertebral discs, including dehydration, decreased disc volume and decreased proteoglycan content (29,30). Consequently, $\mathrm{T}_{1 \rho}, \mathrm{T}_{2}$ and ADC values, which may be influenced by the disc matrix composition, should be strongly related to age. The present results indicated negative associations of $T_{1 \rho}, T_{2}$ and ADC values were exhibited with age, which was in line with previous findings $(10,13,16,31,32)$. Furthermore, $T_{1 \rho}$ values exhibited the weakest correlation with age (which remained stable $<45$ years), whereas $\mathrm{T}_{2}$ and ADC values exhibited stronger correlations with age (the $T_{2}$ values were increased slightly and the ADC values were increased remarkably over the age range of 45-50 years; and both values were rapidly increased after the age of 50 years). These results indicated the relative stability of the $T_{1 \rho}$ measurement and suggested that the changes in proteoglycan content were less severe below the age of 45 years. These findings are consistent with previous results, which demonstrated that the repair process of the nucleus pulposus matrix is difficult after the age of 50 years (33). Conversely, the decrease in $\mathrm{T}_{2}$ value is primarily correlated with water loss (12). Unexpectedly, the ADC values were dramatically increased during the age range of 45-50 years. When combined with color-coded mapping, in the normal nucleus pulposus, high ADC values were observed in the peripheral area, whereas high $\mathrm{T}_{1 \rho}$ and $\mathrm{T}_{2}$ values were indicated in the core region. The ADC values are strongly correlated with proteoglycan and water content, which may affect the matrix integrity of the nucleus pulposus (17). Based on prior and present studies, we provided the following hypothesis for the results. Reduced water content was more dominant due to the proteoglycan stability and thus the ADC values were decreased over the age range of below 45 years. In addition, we hypothesize that during the age range of 45-50 years, the proteoglycan levels were gradually diminished and the $T_{1 \rho}$ values were gradually decreased, which resulted in a reduced restriction of water motion. After the compromised matrix integrity resulting from the reduced proteoglycan and water constants may primarily contribute to the declined ADC values (27). Therefore, regarding the correlation between DWI and IDD, subject age should be taken into account for the result analysis $(17,18,20,34)$. Moreover, ROC analysis suggested that the nucleus pulposus composition may change dramatically during the age range of 45-50 years and IDD development may accelerate after the age of 50 years. These findings may contribute to determining the optimum timing for intervention and treatment of the disease. In addition, the present study indicated a positive correlation between the $\mathrm{T}_{1 \rho}, \mathrm{T}_{2}$ and ADC values, particularly between the $\mathrm{T}_{2}$ and ADC values. These three indicators were decreased progressively and exhibited similar patterns following the degeneration in nucleus pulposi.

The present study exhibited some limitations. The first limitation is the lack of a gold standard for the IDD grading, particularly with the biochemical and histological methods. Although the Pfirrmann grading system exhibited excellent agreement, this method only provided a morphological and semi-quantitative evaluation of IDD in vivo. Additional in vitro examination is required to fully elucidate these issues. Other limitations include the loss of MRI signals and the bias due to the physiological motion. Moreover, banding artifacts were inevitable and may have influenced the $\mathrm{T}_{1 p}$ signals. Another limitation is the limited sample size, particularly for the subjects within the age range of 45-50 years (only 20 discs). Therefore, further studies with large sample sizes are necessary.

In conclusion, the present results indicated that $T_{1 \rho}$ values of the nucleus pulposi at L4/5 and L5/S1 were significantly reduced compared with the values at L1/2, L2/3 and L3/4, whereas no significant differences were observed in $\mathrm{T}_{2}$ values. ADC values of L1/2 were significantly decreased from $\mathrm{L} 2 / 3$ and $\mathrm{L} 3 / 4$ and $\mathrm{T}_{1 \rho}, \mathrm{T}_{2}$ and ADC values were positively correlated with each other. $T_{1 \rho}$ values were significantly different between each grade (with the exception of grades IV and V); and the $\mathrm{T}_{2}$ and ADC values were not significantly different between grades I, II, IV, and grade $\mathrm{V}$. Moreover, the $\mathrm{T}_{1 \rho}, \mathrm{T}_{2}$ and ADC values were significantly decreased with the increase of age. Our findings suggest that $T_{1 \rho}$ and $T_{2}$ mapping and DWI are promising techniques for the in vivo diagnosis of IDD. Furthermore, we conclude that these techniques are particularly useful in determining the appropriate prevention and treatment options for the disease. 


\section{Acknowledgements}

This work was supported by the Medical and Health Technology Project of Guangzhou (grant nos. 20141A011008 and 20151A011075)

\section{References}

1. Schwarzer AC, Aprill CN, Derby R, Fortin J, Kine G and Bogduk N: The relative contributions of the disc and zygapophyseal joint in chronic low back pain. Spine (Phila Pa 1976) 19: 801-806, 1994.

2. Cheung KM, Karppinen J, Chan D, Ho DW, Song YQ, Sham P, Cheah KS, Leong JC and Luk KD: Prevalence and pattern of lumbar magnetic resonance imaging changes in a population study of one thousand forty-three individuals. Spine (Phila $\mathrm{Pa}$ 1976) 34: 934-940, 2009.

3. de Schepper EI, Damen J, van Meurs JB, Ginai AZ, Popham M, Hofman A, Koes BW and Bierma-Zeinstra SM: The association between lumbar disc degeneration and low back pain: The influence of age, gender, and individual radiographic features. Spine (Phila Pa 1976) 35: 531-536, 2010.

4. Kalson NS, Richardson S and Hoyland JA: Strategies for regeneration of the intervertebral disc. Regen Med 3: 717-729, 2008.

5. Masuda K: Biological repair of the degenerated intervertebral disc by the injection of growth factors. Eur Spine J 17 (Suppl 4): S441-S451, 2008.

6. Pfirrmann CW, Metzdorf A, Zanetti M, Hodler J and Boos N: Magnetic resonance classification of lumbar interevertebral disc degeneration. Spine (Phila Pa 1976) 26: 1873-1878, 2001.

7. Griffith JF, Wang YX, Antonio GE, Choi KC, Yu A, Ahuja AT and Leung PC: Modified pfirrmann grading system for lumbar intervertebral disc degeneration. Spine (Phila Pa 1976) 32: E708-E712, 2007.

8. Zuo J, Joseph GB,Li X,Link TM, Hu SS, Berven SH, Kurhanewitz J and Majumdar S: In vivo intervertebral disc characterization using magnetic resonance spectroscopy and T1 $\rho$ imaging: Association with discography and Oswestry Disability Index and Short Form-36 Health Survey. Spine (Phila Pa 1976) 37: 214-221, 2012.

9. Zobel BB, Vadalà G, Del Vescovo R, Battisti S, Martina FM, Stellato L, Leoncini E, Borthakur A and Denaro V: T1 $\rho$ magnetic resonance imaging quantification of early lumbar intervertebral disc degeneration in healthy young adults. Spine (Phila Pa 1976) 37: 1224-1230, 2012.

10. Johannessen W, Auerbach JD, Wheaton AJ, Kurji A, Borthakur A, Reddy R and Elliott DM: Assessment of human disc degeneration and proteoglycan content using T1rho-weighted magnetic resonance imaging. Spine (Phila Pa 1976) 31: 1253-1257, 2006.

11. Mulligan KR, Ferland CE: Gawri R, Borthakur A, Haglund L and Ouellet JA: Axial T1 $\rho$ MRI as a diagnostic imaging modality to quantify proteoglycan concentration in degenerative disc disease. Eur Spine J 24: 2395-2401, 2015.

12. Marinelli NL, Haughton VM, Muñoz A and Anderson PA: T2 relaxation times of intervertebral disc tissue correlated with water content and proteoglycan content. Spine (Phila Pa 1976) 34: 520-524, 2009

13. Ludescher B, Effelsberg J, Martirosian P, Steidle G, Markert B, Claussen $\mathrm{C}$ and Schick F: T2- and diffusion-maps reveal diurnal changes of intervertebral disc composition: An in vivo MRI study at 1.5 Tesla. J Magn Reson Imaging 28: 252-257, 2008.

14. Perry J, Haughton V, Anderson PA, Wu Y, Fine J and Mistretta C: The value of T2 relaxation times to characterize lumbar intervertebral disks: Preliminary results. AJNR Am J Neuroradiol 27: 337-342, 2006.

15. Trattnig S, Stelzeneder D, Goed S, Reissegger M, Mamisch TC Paternostro-Sluga T, Weber M, Szomolanyi P and Welsch GH: Lumbar intervertebral disc abnormalities: Comparison of quantitative T2 mapping with conventional. MR at 3.0 T. Eur Radiol 20 2715-2722, 2010.

16. Marinelli NL, Haughton VM and Anderson PA: T2 relaxation times correlated with stage of lumbar intervertebral disk degeneration and patient age. AJNR Am J Neuroradiol 31: 1278-1282, 2010.
17. Antoniou J, Demers CN, Beaudoin G, Goswami T, Mwale F, Aebi M and Alini M: Apparent diffusion coefficient of intervertebral discs related to matrix composition and integrity. Magn Reson Imaging 22: 963-972, 2004

18. Kealey SM, Aho T, Delong D, Barboriak DP, Provenzale JM and Eastwood JD: Assessment of apparent diffusion coefficient in normal and degenerated intervertebral lumbar disks: Initial experience. Radiology 235: 569-574, 2005.

19. Kerttula L, Kurunlahti M, Jauhiainen J, Koivula A, Oikarinen J and Tervonen O: Apparent diffusion coefficients and T2 relaxation time measurements to evaluate disc degeneration. A quantitative MR study of young patients with previous vertebral fracture. Acta Radiol 42: 585-591, 2001.

20. Niu G, Yang J, Wang R, Dang S, Wu EX and Guo Y: MR imaging assessment of lumbar intervertebral disk degeneration and age-related changes: Apparent diffusion coefficient versus T2 quantitation. AJNR Am J Neuroradiol 32: 1617-1623, 2011.

21. Wang YX, Zhao F, Griffith JF, Mok GS, Leung JC, Ahuja AT and Yuan J: T1rho and T2 relaxation times for lumbar disc degeneration: An in vivo comparative study at 3.0-Tesla MRI. Eur Radiol 23: 228-234, 2013.

22. Nagashima M, Abe H, Amaya K, Matsumoto H, Yanaihara $H$, Nishiwaki Y, Toyama Y and Matsumoto M: A method for quantifying intervertebral disc signal intensity on T2-weighted imaging. Acta Radiol 53: 1059-1065, 2012.

23. Auerbach JD, Johannessen W, Borthakur A, Wheaton AJ, Dolinskas CA, Balderston RA, Reddy R and Elliott DM: In vivo quantification of human lumbar disc degeneration using T (1rho)-weighted magnetic resonance imaging. Eur Spine J 15 (Suppl 3): S338-S344, 2006.

24. Blumenkrantz G, Zuo J, Li X, Kornak J, Link TM and Majumdar S: In vivo 3.0-tesla magnetic resonance T1rho and T2 relaxation mapping in subjects with intervertebral disc degeneration and clinical symptoms. Magn Reson Med 63: 1193-1200, 2010.

25. Regatte RR, Akella SV, Borthakur A, Kneeland JB and Reddy R: Proteoglycan depletion-induced changes in transverse relaxation maps of cartilage: Comparison of T2 and T1rho. Acad Radiol 9: 1388-1394, 2002

26. Xia Y, Farquhar T, Burton-Wurster N and Lust G: Origin of cartilage laminae in MRI. J Magn Reson Imaging 7: 887-894, 1997.

27. Martin JT, Collins CM, Ikuta K, Mauck RL, Elliott DM, Zhang Y, Anderson DG, Vaccaro AR, Albert TJ, Arlet V and Smith HE: Population average T2 MRI maps reveal quantitative regional transformations in the degenerating rabbit intervertebral disc that vary by lumbar level. J Orthop Res 33: 140-148, 2015.

28. Buckwalter JA: Aging and degeneration of the human intervertebral disc. Spine (Phila Pa 1976) 20: 1307-1314, 1995

29. Erwin WM, DeSouza L, Funabashi M, Kawchuk G, Karim MZ, Kim S, Mädler S, Matta A, Wang X and Mehrkens KA: The biological basis of degenerative disc disease: Proteomic and biomechanical analysis of the canine intervertebral disc. Arthritis Res Ther 17: 240, 2015.

30. Hanaei S, Abdollahzade S, Khoshnevisan A, Kepler CK and Rezaei N: Genetic aspects of intervertebral disc degeneration. Rev Neurosci 26: 581-606, 2015.

31. Nguyen AM, Johannessen W, Yoder JH, Wheaton AJ, Vresilovic EJ, Borthakur A and Elliott DM: Noninvasive quantification of human nucleus pulposus pressure with use of T1rho-weighted magnetic resonance imaging. J Bone Joint Surg Am 90: 796-802, 2008.

32. Blumenkrantz G, Li X, Han ET, Newitt DC, Crane JC, Link TM and Majumdar S: A feasibility study of in vivo T1rho imaging of the intervertebral disc. Magn Reson Imaging 24: 1001-1007, 2006.

33. Siemionow K, An H, Masuda K, Andersson G and Cs-Szabo G: The effects of age, sex, ethnicity, and spinal level on the rate of intervertebral disc degeneration: A review of 1712 intervertebral discs. Spine (Phila Pa 1976) 36: 1333-1339, 2011.

34. Niinimäki J, Korkiakoski A, Ojala O, Karppinen J, Ruohonen J, Haapea M, Korpelainen R, Natri A and Tervonen O: Association between visual degeneration of intervertebral discs and the apparent diffusion coefficient. Magn Reson Imaging 27: 641-647, 2009. 